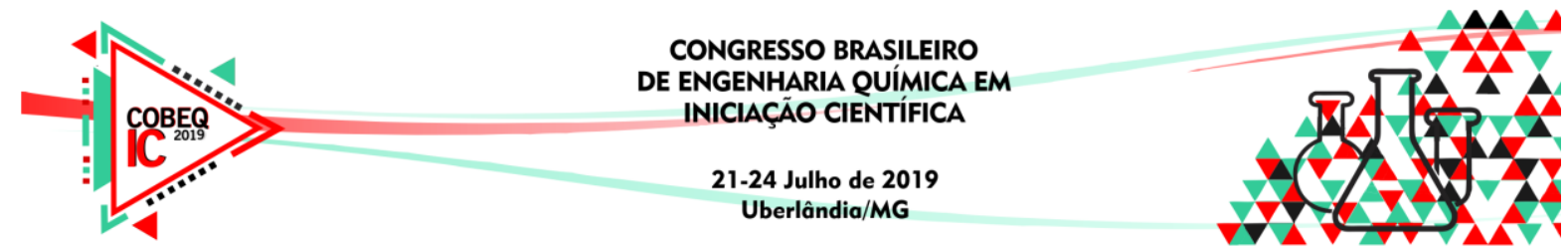

\title{
CARBONIZAÇÃO HIDROTÉRMICA DOS RESÍDUOS DE ACEROLA (Malphigia emarginata D.C.): INFLUÊNCIA DAS VARIÁ VEIS DE PROCESSO
}

\author{
B. A. SOUZA ${ }^{1}$, G. A. N. BARBOSA ${ }^{1}$, G. D. R. NOGUEIRA ${ }^{1}$, C. R. DUARTE ${ }^{1}$, M. A. S. \\ BARROZO $^{1}$ \\ ${ }^{1}$ Universidade Federal de Uberlândia, Faculdade de Engenharia Química \\ E-mail para contato: masbarrozo@ufu.br
}

\begin{abstract}
RESUMO - A carbonização hidrotérmica (HTC) é uma técnica promissora para o melhor gerenciamento e melhor aproveitamento de resíduos agroindustriais. Neste estudo, investigou-se os efeitos da temperatura, tempo de reação, razão biomassa / água, $\mathrm{pH}$ da água de alimentação e velocidade de agitação na HTC de resíduos de acerola. Os efeitos dessas variáveis sobre o rendimento do processo, os grupos funcionais oxigenados totais de hidrocarvões e da área superficial foram quantificados. Observou-se que os rendimentos obtidos variaram entre $53 \%$ a $64 \%$. Os grupos funcionais oxigenados atingiram um máximo de $7,4 \mathrm{mmol} / \mathrm{g}$. Os resultados indicaram que todas as variáveis foram estatisticamente significativas $(\mathrm{p}<0,05)$ para a área superficial, enquanto apenas o $\mathrm{pH}$ da água de alimentação e a agitação foram significativos para os grupos funcionais oxigenados. Os resultados indicaram que a carbonização hidrotérmica dos resíduos de acerola pode ser uma tecnologia sustentável para a modificação de resíduos subutilizados e sua aplicação como adsorventes de contaminantes ambientais.
\end{abstract}

\section{INTRODUÇÃO}

A geração de resíduos em países com grande produção agrícola, como o Brasil, é uma crescente preocupação ambiental. A destinação destes resíduos não deve ser negligenciada e pode-se utilizá-lo como fonte alternativa para produção de bioenergia e materiais diversos (Sabio et al., 2016). O Brasil se destaca no cultivo de frutas tropicas, como a acerola. Grandes quantidades de resíduos desta fruta são geradas, pois a produção nacional é a maior do mundo e a acerola é consumida, principalmente, por meio de produtos processados (Silva et al., 2016).

Uma alternativa para a reutilização do resíduo de acerola é através da carbonização hidrotérmica (HTC). A HTC é uma técnica que se destaca dentre os processos de carbonização e pode ser usada para a produção de adsorventes, tendo como matéria prima, por exemplo, os resíduos de frutas. Esta técnica tem como produto principal o hidrocarvão, que pode ser utilizado como combustíveis, corretores de solo, adsorventes, entre outros (Hu et al., 2014).

A HTC é realizada em meio aquoso em um recipiente fechado a altas temperaturas e pressões autógenas. Nestas condições, as ligações de hidrogênio perdem a força, mudando a 


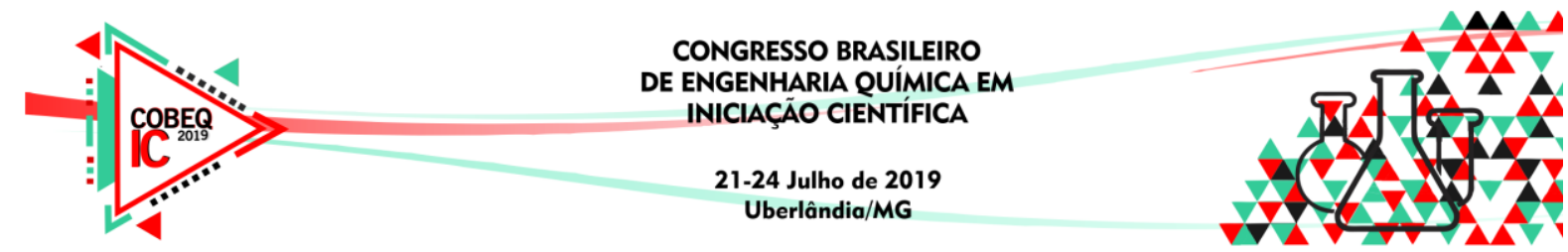

constante dielétrica e a polaridade da água. Assim a água passa a fazer parte das reações, atuando como catalisador. Em condições normais tal caminho reacional não aconteceria. Durante esse processo, a biomassa é decomposta, o fato de a água agir como catalisador e a matéria prima úmida ser convertida em produto sólido, causa uma diminuição no uso de energia, evitando a necessidade de a matéria prima ser seca antes do processo. (Libra et al., 2011).

Este trabalho tem por objetivo analisar como a temperatura, tempo de residência, concentração (razão biomassa/água), $\mathrm{pH}$ do meio e velocidade de agitação influenciam no rendimento de sólidos (SY), nos grupos oxigenados totais (GOT) e na área superficial do carvão, interpretada pelo método de Barrett-Emmett-Teller $\left(\mathrm{S}_{\mathrm{BET}}\right)$.

\section{MATERIAL E MÉTODOS}

\subsection{Material Percursor}

Os resíduos da acerola foram disponibilizados pela indústria de processamentos Fruteza, localizada em Dracena - SP. O resíduo foi seco a $105^{\circ} \mathrm{C}$, em estufa por $24 \mathrm{~h}$. Após isso foi triturado e selecionado a biomassa cujo a granulometria estava entre 0,125 e 2,00 mm.

\subsection{Aparato e Procedimento Experimental}

O processo de carbonização hidrotérmica (HTC) foi realizado em um reator de aço inoxidável, com $60 \mathrm{~mm}$ de diâmetro e $80 \mathrm{~mm}$ de altura, com manômetro e termopar do tipo K acoplados. Utilizou-se um agitador magnético com o propósito de controlar e variar a taxa de agitação, a qual foi aferida através de filmagens em uma câmera de alta velocidade com 500 quadros por segundo. Para o aquecimento, um sistema de resistência com coleira com um controlador proporcional-integral-derivativo - PID foi empregado. A pressão foi autogerada pelo sistema e não foi controlada. Após o tempo de reação escolhido, o reator teve seu sistema desligado e então foi resfriado e despressurizado. O produto sólido foi filtrado e depois seco em estufa a $105^{\circ} \mathrm{C}$ por $24 \mathrm{~h}$.

Os testes foram realizados analisando cinco variáveis diferentes: temperatura - $\mathrm{T}\left({ }^{\circ} \mathrm{C}\right)$, tempo - t (h), razão de biomassa/água $-\mathrm{R}(\%), \mathrm{pH}$ da água de alimentação $-\mathrm{pH}(-)$ e velocidade de agitação - $\omega(\mathrm{rpm})$, o set point dessas variáveis para cada experimento seguiu conforme planejamento apresentando na Tabela 1. Como resposta foram considerados o rendimento sólido (SY), os grupos oxigenados totais (GOT) e a área superficial pelo método de Barrett-Emmett-Teller (SBET).

SY foi calculado usando a Equação 1:

$$
Y(\%)=\frac{m_{H C}}{m_{B I O}} \times 100
$$

Onde: $m_{H C}$ é a massa de hidrocarvão e $m_{B I O}$ secos é a massa de matéria-prima de resíduos de acerola. 


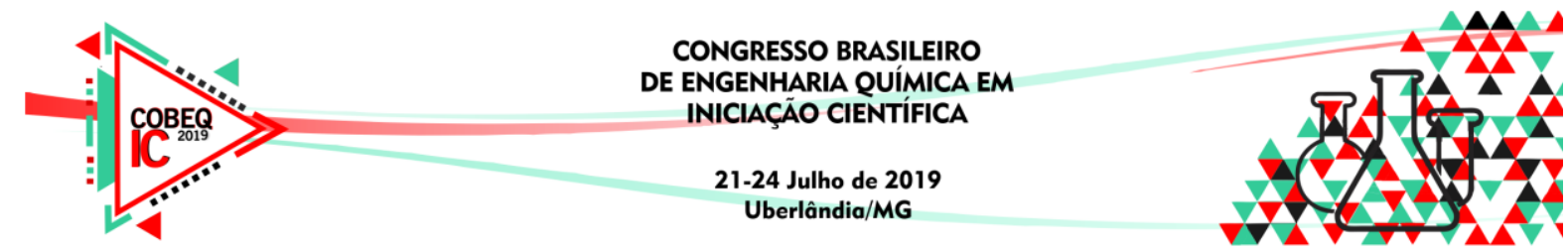

Tabela 1 - Planejamento experimental da carbonização hidrotérmica da biomassa da acerola

\begin{tabular}{|c|c|c|c|c|c|}
\hline Experimento & $\mathrm{T}\left({ }^{\circ} \mathrm{C}\right)$ & $\mathrm{t}(\mathrm{h})$ & $\mathrm{R}(\%)$ & $\mathrm{pH}(-)$ & $\omega(\mathrm{rpm})$ \\
\hline 1 & 154 & 5,00 & 7,00 & 7,00 & 1500,00 \\
\hline 2 & 206 & 5,00 & 7,00 & 7,00 & 1500,00 \\
\hline 3 & 180 & 0,69 & 7,00 & 7,00 & 1500,00 \\
\hline 4 & 180 & 9,31 & 7,00 & 7,00 & 1500,00 \\
\hline 5 & 180 & 5,00 & 1,83 & 7,00 & 1500,00 \\
\hline 6 & 180 & 5,00 & 12,17 & 7,00 & 1500,00 \\
\hline 7 & 180 & 5,00 & 7,00 & 1,84 & 1500,00 \\
\hline 8 & 180 & 5,00 & 7,00 & 12,2 & 1500,00 \\
\hline 9 & 180 & 5,00 & 7,00 & 7,00 & 446,37 \\
\hline 10 & 180 & 5,00 & 7,00 & 7,00 & 2553,63 \\
\hline
\end{tabular}

\subsection{Caracterização dos Hidrocarvões e Tratamento Estatístico}

Titulação de Boehm: A quantidade de grupos funcionais oxigenados (GOT) na superfície do hidrocarvão foram determinados através da titulação de Boehm (Sun et al. 2015) com diferentes soluções $\left(\mathrm{NaOH}, \mathrm{Na}_{2} \mathrm{CO}_{3}\right.$ e $\left.\mathrm{NaHCO}_{3}\right)$.

$\underline{S}_{\mathrm{BET}}:$ As áreas superficiais das amostras foram determinadas utilizando o equipamento ASAP 2020 (Micromeritics), com experimentos de adsorção de nitrogênio realizados a $77 \mathrm{~K} \mathrm{e}$ interpretados pelo método de Barrett-Emmett-Teller (BET).

Todos os resultado da caracterização dos hidrocarvões foram expressos como média \pm desvio padrão. Diferenças significativas $(\mathrm{p}<0,05)$ entre as médias foram avaliada por análise de variância (ANOVA) e Teste de Tukey.

\section{RESULTADOS E DISCUSSÕES}

Conforme planejamento experimental, os resultados para o rendimento dos hidrocarvões (SY) e os grupos funcionais oxigenados (GOT) são apresentados na Figura 1. Observa-se, na Figura 1-a que os rendimentos obtidos variaram entre 53\% a 64\%, aproximadamente. Tais valores são similares aos rendimentos de sólidos em carbonização hidrotérmica de resíduos agroindustriais observados por outros autores. Sabio et al. (2016) encontraram rendimentos de até $62,96 \%$ para resíduos da casca de tomate no processo de HTC.

Ao analisar a Figura 1-a, observa-se que a temperatura (T), o tempo de residência (t) e a razão de biomassa/água $(\mathrm{R})$ são as variáveis que mais influenciaram a variação de $\mathrm{SY}$. Maiores $\mathrm{T}$ e $\mathrm{t}$ potencializam vários mecanismos de degradação como desidratação, descarboxilação e desmetanização. A temperatura ainda pode promover a miscibilidade de alguns compostos orgânicos da biomassa na água do meio reacional e diminuir a viscosidade do líquido, permitindo que haja uma maior penetração na matriz da biomassa, consequentemente, facilitando a sua degradação (Chen et al., 2018). O aumento em $\mathrm{R}$ 


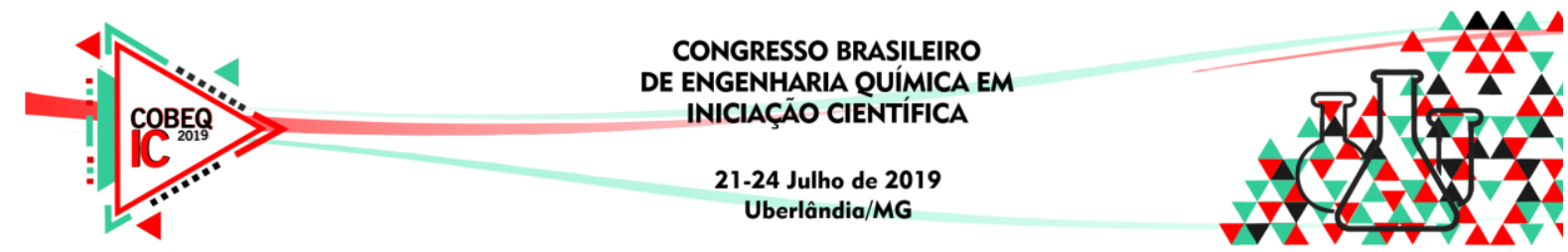

(experimentos 5 e 6) apresentou efeito positivo, i.e., com a variação positiva de $\mathrm{R}$ também houve um maior SY. Sabio et al. (2016) também observaram o efeito positivo para R na carbonização hidrotérmica de resíduo de tomate.

Figura 1 - Rendimento de sólidos (SY, a) e total de grupos funcionais oxigenados (OGF, b) obtidos para cada experimento.

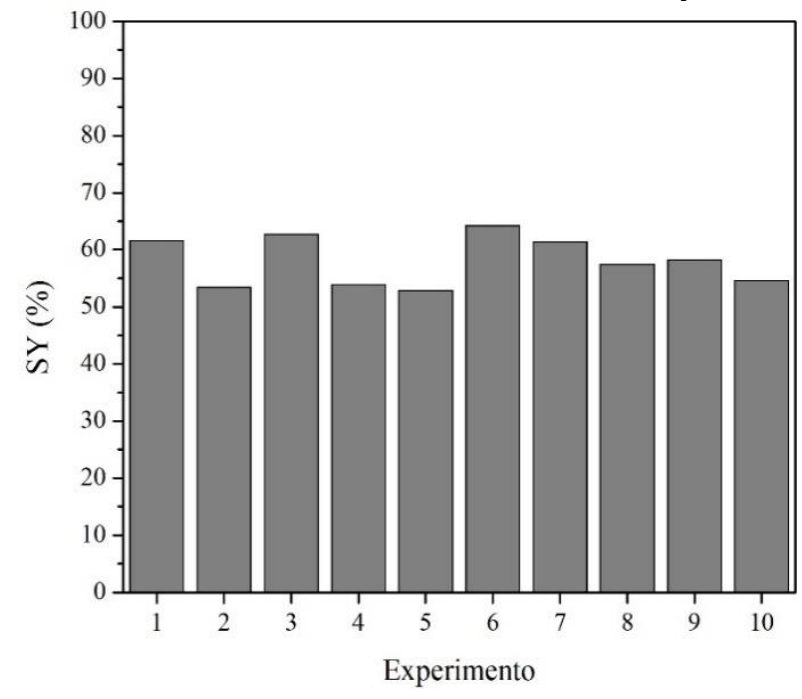

(a)

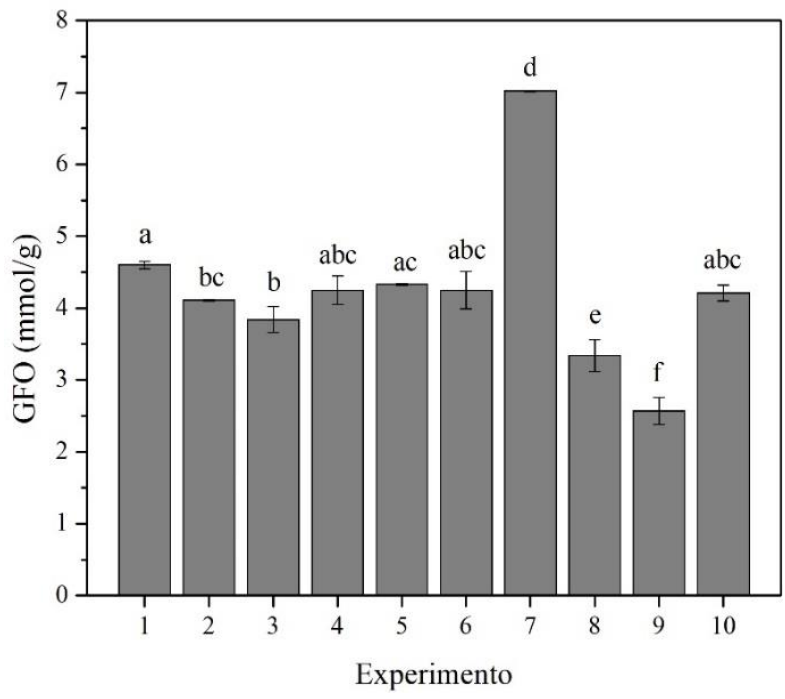

(b)

Os efeitos do $\mathrm{pH}$ da água de alimentação $(\mathrm{pH})$ e velocidade de agitação $(\omega)$ em $\mathrm{SY}$ são menores que os das outras variáveis. Vários componentes da biomassa podem ser vulneráveis à degradação em diferentes níveis de pH (Reza et al., 2015). O cálculo do SY considera apenas a massa final do sólido e desconsidera a variação de massa de cada componente, sendo que em meios mais ou menos ácidos pode haver mais ou menos degradação de um determinado componente (Reza et al., 2015). Também se sabe que o pH da água de alimentação influencia as mudanças na via de reação (Yin et al., 2011). Já a ligeira redução de SY promovida pela agitação pode estar relacionada com o aumento de colisões do material auxiliando na sua degradação (Du; Chen; Lucas, 2014).

A Figura 1-b mostra os resultados encontrados para os grupos funcionais oxigenados (GFO), os quais incluem os grupos carboxílicos $(-\mathrm{COOH})$, lactônicos $(-\mathrm{C}=\mathrm{O})$ e os fenólicos ($\mathrm{OH})$. Percebe-se que GOT total variou entre 2,5 e 7,4 mmol/g de massa seca. Os efeitos das variáveis estudadas nos GOT foram diferentes daqueles observados no SY. O pH e a $\omega$ foram os que tiveram maiores efeitos sobre GOT e os efeitos de T, t e R não foram estatisticamente significativos $(\mathrm{p}<0,05)$.

Zhao et al. (2018), também observaram que meios mais ácidos levaram à valores mais altos de GOT. Portanto, maiores velocidades de agitação levaram a maiores conversões de reações de formação de GOT (Li et al., 2018). Sabe-se ainda que quanto maior a quantidade de grupos funcionais oxigenados tem-se uma maior quantidade de sítios ácidos, melhorando a capacidade de sorção dos hidrocarvões (Sun et al, 2015).

O Tabela 2, mostra os resultados encontrados para a área superficial do hidrocarvão, pelo método BET. Constata-se que todas as variáveis analisadas tiveram significativa 


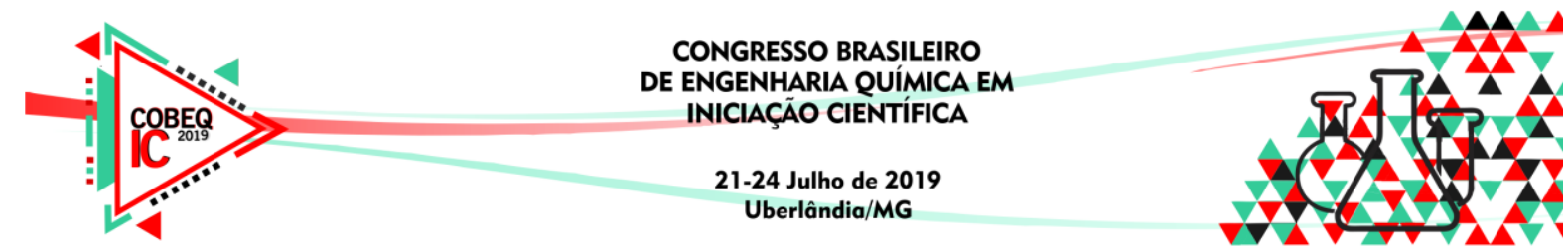

importância na variação da área superficial do hidrocarvão. T foi a variável que se mostrou

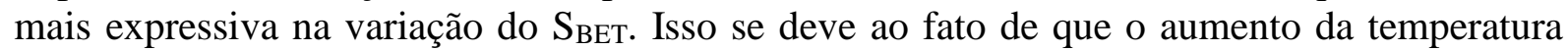
causa uma remoção dos compostos voláteis (Almeida et al., 2017). Desta forma, um tempo de residência maior em uma temperatura amena, permite que as reações de remoção ocorram completamente.

Tabela 2 - Valores de $\mathrm{S}_{\text {BET }}$ para cada experimento

\begin{tabular}{|c|c|}
\hline Experimento & SBET $\left(\mathbf{m}^{2} / \mathbf{g}\right)$ \\
\hline 1 & $5.20 \pm 0.06^{\mathrm{a}}$ \\
\hline 2 & $13.82 \pm 0.04^{\mathrm{b}}$ \\
\hline 3 & $1.15 \pm 0.06^{\mathrm{c}}$ \\
\hline 4 & $6.86 \pm 0.07^{\mathrm{d}}$ \\
\hline 5 & $3.41 \pm 0.10^{\mathrm{e}}$ \\
\hline 6 & $5.13 \pm 0.06^{\mathrm{a}}$ \\
\hline 7 & $9.39 \pm 0.09^{\mathrm{f}}$ \\
\hline 8 & $10.25 \pm 0.08^{\mathrm{g}}$ \\
\hline 9 & $9.61 \pm 0.05^{\mathrm{h}}$ \\
\hline 10 & $7.22 \pm 0.09^{\mathrm{i}}$ \\
\hline
\end{tabular}

Os hidrocarvões apresentam valores de $\mathrm{S}_{\mathrm{BET}}$ relativamente pequenos em comparação a outros materiais adsorventes cujas áreas são, geralmente, maiores que $800 \mathrm{~m}^{2} / \mathrm{g}$ (Zhou et al., 2017). No entanto, muitos pesquisadores relataram que um fator chave na adsorção de muitos poluentes é a ocorrência de ligações químicas entre grupos funcionais e adsorventes, o que torna a área de superfície adsorvente e a estrutura porosa menos importante (Zhao et al., 2018).

\section{CONCLUSÃO}

Foi analisado, neste trabalho, a influência das variáveis temperatura, concentração, tempo de detenção, $\mathrm{pH}$ e velocidade de agitação no rendimento (SY) da carbonização hidrotérmica da biomassa da acerola. No geral, todas as variáveis analisadas demonstraram algum grau de influência no SY, com destaque para a temperatura e tempo de residência. E também influenciam no GOT, mas neste destacam-se o pH e velocidade de agitação. Já para o $\mathrm{S}_{\mathrm{BET}}$, a variável que se mostrou mais relevante foi a temperatura. Deste modo, constatou-se quais as principais variáveis precisam ser controladas para obtenção de um melhor hidrocarvão.

\section{REFERÊNCIAS}

ALMEIDA, V.C.; RONIX, A.; PEZOTI, O.; SOUZA, L.S.; SOUZA, I.P.A.F.; BEDIN, K.C.; SOUZA, P.S.C; SILVA, T.L.; MELO, S.A.R; CAZETTA, A.L. Hydrothermal carbonization of coffee husk: Optimization of experimental parameters and adsorption of methylene blue dye. J. Environ. Chem. Eng, v. 5, p.4841-4849, 2017. 


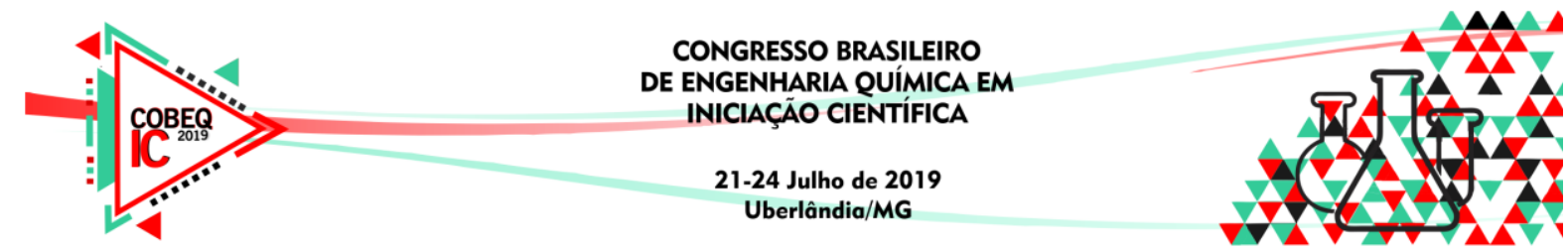

CHEN, X.; MA, X.; PENG, X.; LIN, Y.; YAO, Z. Conversion of sweet potato waste to solid fuel via hydrothermal carbonization. Bioresour. Technol., v. 249, p. 900-907, 2018.

DU, SHAN-WEN; CHEN, WEI-HSIN; LUCAS, JOHN A. Pretreatment of biomass by torrefaction and carbonization for coal blend used in pulverized coal injection. Bioresour. Technol., v. 161, p.333-339, 2014.

HU, J.; SHEN, D.; WU, S., ZHANG, H.; XIAO, R. "Effect of temperature on structure evolution in char from hydrothermal degradation of lignin", J. Anal. Apll. Pyrolysis, v. 106, p. 118-124, 2014.

LI, Y.; XIAO, K.; LIU, H.; YI, L.; ZHANG, X.; HU, H.; YAO, H. Correlations between hydrochar properties and chemical constitution of orange peel waste during hydrothermal carbonization. Bioresour. Technol., v. 265, p. 432-436, 2018.

LIBRA, J. A.; RO, K. S.; KAMMANN. C.; FUNKE, A.; BERGE,N. D.; NEUBAUER, Y.; TITIRICI, M. M.; FÜHNER, C.; BENS, O.; KERN, J.; EMMERICH, K. H. Hydrothermal carbonization of biomass residuals: a comparative review of the chemistry, process and applications of wet and dry pyrolisis. Biofuels, v. 2, p. 71-106, 2011.

REZA, M. T.; ROTTLER, E.; HERKLOTZ, L.; WIRTH, B. Hydrothermal carbonization (HTC) of wheat straw: Influence of feedwater $\mathrm{pH}$ prepared by acetic acid and potassium hydroxide. Bioresour. Technol., v. 182, p. 336-344, 2015.

SABIO, E.; ÁlVAREZ-MURILlO, A.; ROMÁN, S.; LEDESMA, B. "Conversion of tomato-peel waste into solid fuel by hydrothermal carbonization: Influence of the processing variables", Waste Manag., v. 47, p. 122-132, 2016.

SILVA, P.B.; DUARTE, C.R.; BARROZO, M.A.S. "Dehydration of acerola (Malpighia emarginata D.C.) residue in a new designed rotary dryer: Effect of process variables on main bioactive compounds", Food Bioprod. Process., v. 98, p. 62-70, 2016.

SUN, K.; TANG, J.; GONG, Y.; ZHANG, H. Characterization of potassium hydroxide $(\mathrm{KOH})$ modified hydrochars from different feedstocks for enhanced removal of heavy metals from water. Environ. Sci. Pollut. Res., v. 22, p. 16640-16651, 2015.

YIN, S., MEHROTRA, A.K., TAN, Z. Alkaline hydrothermal conversion of cellulose to biooil: Influence of alkalinity on reaction pathway change. Bioresour. Technol., v. 102, p. 6605-6610, 2011.

ZHAO, X.; MA, X.; ZHENG, P. The preparation of carboxylic-functional carbon-based nanofibers for the removal of cationic pollutants. Chemosphere, v. 202, p. 298-305, 2018.

ZHOU, N., CHEN, H., XI, J., YAO, D., ZHOU, Z., TIAN, Y., LU, X. Biochars with excellent $\mathrm{Pb}$ (II) adsorption property produced from fresh and dehydrated banana peels via hydrothermal carbonization. Bioresour. Technol., v. 232, p. 204-210, 2017. 